Proceedings of the

International Geometry Center

Vol. 11, no. 1 (2018) pp. 12-26

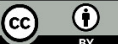

\title{
Trajectory equivalence of optimal Morse flows on closed surfaces
}

\author{
Zlata Kibalko, Alexandr Prishlyak, Roman Shchurko
}

\begin{abstract}
We consider optimal Morse flows on closed surfaces. Up to topological trajectory equivalence such flows are determined by marked chord diagrams. We present list all such diagrams for flows on nonorientable surfaces of genus at most 4 and indicate pairs of diagrams corresponding to the flows and their inverses.
\end{abstract}

Анотація. В роботі досліджуються оптимальні потоки Морса, тобто потоки, що мають мінімальне число особливих точок серед усіх потоків Морса. Під потоком Морса ми розуміємо потік Морса-Смейла без замкнених траєкторій, тобто потік без замкнених траєкторій, зі скінченим числом нерухомих точок, які є гіперболічними, і такий, що гранична множина кожної траєкторії складається з нерухомих точок, а стійкі та нестійкі многовиди нерухомих точок перетинаються трансверсально. Оптимальні потоки Морса характеризуються тим, що мають єдиний стік i єдине джерело i, отже, є полярними потоками. Кожному оптимальному потоку ставиться у відповідність хордова діаграма. При цьому межа регулярного околу джерела розглядається як коло, а стійкім многовидам сідлових точок відповідають хорди. На неорієнтовних поверхнях хорди мають додаткове маркування +1 або -1 . Маркована хордова діаграма $\epsilon$ повним топологічним інваріантом оптимального потоку Морса. Описано процес побудови оберненої хордової діаграми, що відповідає потоку, у якого змінено напрямок руху за траєкторіями на протилежний. Марковані хордові діаграми задають $f$-атоми за Болсіновим і Фоменком. При цьому маркована діаграма і їй обернена задають один і той самий атом.

Складено список всіх маркованих хордових діаграм і знайдені їх обернені для оптимальних потоків на неорієнтованих поверхнях роду не вище 4. Зокрема, на проективні площині існує єдиний, з точністю до траєкторної еквівалентності, оптимальний потік Морса (одна діаграма). На пляшці Клейна існує два таких не еквівалентних потоки. На неорієнтованій поверхні роду 3 існує дві пари взаємно обернених діаграм i 4 діаграми обернених самі собі, а на неорієнтовній поверхні роду 4 існує 12 пар взаємно обернених діаграм та 23 діаграми обернених самі собі.

Keywords: Morse, topological classification, flow, chord diagram 


\section{INTRODUCTION}

The problem of topological classification of objects or structures is one of the main problems in the topology and theory of dynamical systems. Often the class of such objects consists of structurally stable dynamical systems. They are interesting due to the fact that their topological structure does not change under small changes of the parameters by which they are given. Morse-Smale flows on surfaces are examples of structurally stable dynamical systems. There are many papers dealing with their topological classification, for example [2], [3], [9]. An overview of different approaches to the above problem can be found in [6].

To classify Morse functions on surfaces and integrable Hamiltonian systems Bolsinov and Fomenko introduced the concept of atoms and molecules. They can also be used to classify Morse flows [1].

In fact, Morse flows are gradient flows of Morse functions. In this case the atoms specify the associated handle decompositions. If the Morse flow has one source, then the corresponding atom is a marked chord diagram.

The purpose of the present paper is to find all such chord diagrams corresponding to flows with one sink and to determine which of them correspond to reverse flows on nonorientable surfaces of small genus.

\section{Morse-Smale Flows}

We consider $C^{1}$-smooth dynamical systems on a smooth manifold $M$ with continuous time (flows). Since such a flow on a closed manifold is always generated by a smooth vector field, all the concepts that are introduced for dynamical systems will be applied to vector fields.

Let $X_{t}$ the flow generated by a vector field $X$.

Definition 2.1. Fixed (singular) points and closed trajectories of a dynamical system are called critical elements.

Definition 2.2. A singular point $p$ of a vector field $X=\left\{X_{1}, \ldots, X_{n}\right\}$ is called non-degenerate or hyperbolic, if in some local coordinates $\left(x_{1}, \ldots, x_{n}\right)$ at $p$ the Jacobi matrix

$$
\left(\frac{\partial X_{i}}{\partial x_{j}}\right)_{i, j=1}^{n}
$$

does not contain eigenvalues with zero real part (that is eigenvalues on the imaginary axis).

A closed orbit $\gamma$ of $X$ is called hyperbolic whenever the differential of the Poincaré map has no eigenvalues of absolute value 1 at some point $p \in \gamma$. 
Definition 2.3. A stable manifold (denoted by $W^{s}(p)$ or $S(p)$ ) of a critical element $p$ is the following set

$$
S(p)=\left\{x \in M: \lim _{t \rightarrow \infty} X_{t}(x)=p\right\} .
$$

Similarly, the set

$$
U(p)=\left\{x \in M: \lim _{t \rightarrow-\infty} X_{t}(x)=p\right\} .
$$

is called the unstable manifold $\left(W^{u}(p)\right.$ or $\left.U(p)\right)$ of $p$.

Definition 2.4. [7, p.118] A point $p \in M$ is wandering for $X$ if there exists a neighborhood $V$ of $p$ and a number $n>0$ such that $X_{t}(V) \cap V=\emptyset$ for $|t|>n$. Otherwise $p$ is called nonwandering.

The sets of nonwandering points of a vector field $X$ is denoted by $\Sigma(X)$.

Definition 2.5. [7, p.119] A vector field $X$ on compact manifold $M$ is Morse-Smale whenever:

1) $X$ has a finite number of critical elements and all of them are hyperbolic;

2 ) the set of non-wandering points $\Sigma(X)$ coincides with the union of all critical elements of $X$;

3) stable manifolds of critical elements are transversal to unstable manifolds.

Definition 2.6. A fixed point $y \in M$ is called an $\omega$-limit (resp. an $\alpha$-limit) point for $x \in M$ if there exists a sequence $\left\{t_{i}\right\}$ such that $t_{i} \rightarrow+\infty$ (resp. $t_{i} \rightarrow-\infty$ ) and $X_{t_{i}}(x) \rightarrow y$. The set of all $\omega$-limit points (resp. $\alpha$-limit points) of $x$ is called $\omega$-limit ( $\alpha$-limit) set.

Condition 2) of the definition of Morse-Smale dynamical system can be replaced by the following condition

$2)^{\prime}$ for each point $x \in M$ its $\alpha$-limit and $\omega$-limit sets are contained in the union of critical elements.

For vector fields on two-dimensional manifolds there are three types of non-degenerated (hyperbolic) singular points: sinks, sources, and saddles. Condition 3) in this case is equivalent to the statement that there is no trajectories whose $\alpha$-limit and $\omega$-limit sets are saddle points.

Definition 2.7. Morse-Smale flow (vector field) is called Morse flow (vector field) if it does not contain closed trajectories.

A well known results by Smale [11] claims that every Morse vector field is a gradient field of some Morse function with respect to a certain Riemannian metric.

Properties of Morse-Smale dynamical systems described above, as well as some other properties, can be found in the book by J. Palis and V. di Melo [7]. 


\section{TOPOLOGICAL EQUIVALENCE OF DYNAMICAL SYSTEMS}

Definition 3.1. Let $M$ be a $C^{r}$ manifold, $X^{r}(M)$ be the space of $C^{r}$ vector fields endowed with $C^{r}$ topology. Two vector fields $X, Y \in X^{r}(M)$ are called topologically $\left(C^{k}\right)$ equivalent if there exists a homeomorphism ( $C^{k}$ diffemorphism) $h: M \rightarrow M$ that sends each trajectory of $X$ onto a trajectory of $Y$ preserving their orientations.

Theorem 3.2. [7]. Let $h$ be a topological equivalence between the fields $X, Y \in X^{r}(M)$. Then

1) $p \in M$ is a singular point of the field $X$ if and only if $h(p)$ is a singular point of the field $Y$;

2 ) the trajectory $X(p)$ of the field $X$, which passes through the point $p$, is closed if and only if trajectory $Y(h(p))$ is closed;

$3)$ the image of the $\alpha$-limit ( $\omega$-limit) set of the trajectory $X(p)$ under $h$ is $\alpha$-limit ( $\omega$-limit) set of trajectories $Y(h(p))$.

Definition 3.3. [10]. A vector field $X \in X^{r}(M), r>1$, is called structurally stable if there exists a neighborhood $U$ of the field $X$ in $X^{r}(M)$ such that every $Y \in U$ is topologically equivalent to $X$.

It is known that Morse-Smale dynamical systems are structurally stable [8]. Moreover, every structurally stable vector field on a closed twodimensional manifold is Morse-Smale and these fields form an open everywhere dense set in set of all vector fields.

In higher dimensions, there are structurally stable dynamical systems that are not Morse-Smale systems, and they do not constitute everywhere dense subsets in the spaces of all dynamical systems.

Definition 3.4. A complete topological invariant of a dynamical system from a certain class of dynamical systems is a set of quantities (or algebraic objects) that are identical (isomorphic) for topologically equivalent dynamical systems, and distinct (not isomorphic) for non-equivalents systems.

By the topological classification of a certain class of dynamical systems, we (roughly) mean a construction of a complete topological invariant for systems of this class and such that the equivalence (isomorphism) of invariants can be easily verified, as well as the description of all possible values that the invariant can accept.

\section{Optimal Flows on SURFACES}

Hyperbolicity condition on critical elements means that for vector fields on two-dimensional manifolds there are possible three types of non-degenerate singular points: sinks, sources and saddles. 
A Morse flow on a surface is called optimal if it has the least number of singularities among all Morse flows on this surface.

Theorem 4.1. A Morse flow on a surface is optimal if and only if it contains only one sinks and one sources.

Proof. To prove the theorem, we should show the following statements:

1) a Morse flow on a surface contains at least one sink and one source;

2) there exists Morse flow on a given surface, which contains only one sink and one source;

3 ) if the flow has more than one source or sink, than it is not optimal.

Firstly, It follows from the definition of Morse flow that every trajectory starts at a singular point. As there are only finitely many saddle points, and no more than 2 trajectories could be obtained from each of them, we see that only a finite number of trajectories starts at saddle points. Consequently, the remaining trajectories (it is infinite number) start at the source. By similar arguments there must exist at least one sink.

Secondly. As is well known (see for example [5]), each closed surface is diffeomorphic to a sphere with handles or a sphere with Mobius strips pasted into it. Therefore, it is sufficient to construct a vector field with one sink and one source on these surfaces. As an example of this field, one can take the gradient field of an optimal Morse function (that is, a function with one local minimum and maximum whose existence in is proved in Morse's theory [5]).

Thirdly. By Poincare-Hopf theorem, the Euler characteristic of the surface $M$ is equal to the number of sources plus the number of sinks minus the number of saddle points. This formula shows that increasing the number of sources or sinks leads to increasing of the number of saddle points on a given surface, and, consequently, implies an increasing of the total number of singular points. Hence a flow with one source and one sink is optimal.

Recall that a flow with one source and one sink, also, is called polar. So, optimal flows are precisely polar flows.

\section{Chord diagrams}

Chord diagrams are widely used in modern low-dimension topology, especially in knot theory.

Definition 5.1. A configuration consisting of a circle, $2 n$ distinct points on it, and $n$ chords, which specify a partition of these points into pairs, will be called a chord diagram with $n$ chords, or $n$-diagram. 
Thus, a chord diagram is a graph of valency 3 with a distinguished cycle containing each vertex exactly once. In this case, that cycle is called a circle, and the edges that do not belong to it are called chords.

Definition 5.2. Two chord diagrams are said to be equivalent if there is a homeomorphism between them that maps the cycle into a cycle, and chords into chords.

Let $D$ be an arbitrary chord diagram with $2 n$ points which we enumerate with numbers from 1 to $2 n$ in the clockwise direction starting from a certain fixed point on the dual graph. We construct a substitution $\alpha$ obtained as a result of the transposition multiplication $\left(a_{j}, b_{j}\right), j=1, \ldots, n$, where $a_{j}, b_{j}$ are the numbers of points connected by chord on the diagram $D$ :

$$
\alpha=\left(a_{1}, b_{1}\right)\left(a_{2}, b_{2}\right) \ldots\left(a_{n}, b_{n}\right) .
$$

A change of a starting point can lead to different substitutions. For example, the following substitutions

$$
\alpha_{1}=(1,6)(2,8)(3,7)(4,5) \quad \text { and } \quad \alpha_{2}=(1,7)(2,6)(3,4)(5,8)
$$

correspond to the same chord diagram.

Let $Q$ be a regular polygon in a complex plane $\mathbb{C}$ with $2 n$ vertices being roots of unit of degree $2 n$. Then one can draw (with self intersections) each $2 n$-diagram $D(\alpha)$ in $\mathbb{C}$ by connecting the corresponding pairs of vertices by straight segments and regarding $Q$ as a circle of a diagram. Such a representation of a $2 n$-diagram will be called good.

Obviously, in this approach, each diagram $D(\alpha)$ unequivocally determines the substitution of $\alpha$ and vice versa.

Definition 5.3. Good diagrams $D_{1}$ and $D_{2}$ are called isomorphic if there is a rotation of a circle mapping one diagram into another.

Diagrams $D_{1}$ i $D_{2}$ are equivalent if they are either isomorphic, or $D_{1}$ is isomorphic to a mirror image of $D_{2}$.

There are two non-isomorphic and non-equivalent chord diagrams with two chords:

1) a diagram with intersecting chords; it corresponds to the substitution $(1,3)(2,4)$;

2) a diagram with non-intersecting chords; it corresponds to the substitution $(1,2)(3,4)$.

\section{Chord diagram of an optimal Morse vector field}

Let $X$ be an optimal Morse vector field. Consider some small neighborhood $U$ for the source $x_{0}$ such that its boundary $\partial U\left(x_{0}\right)$ is a smooth curve and transversal to each flow trajectory. 

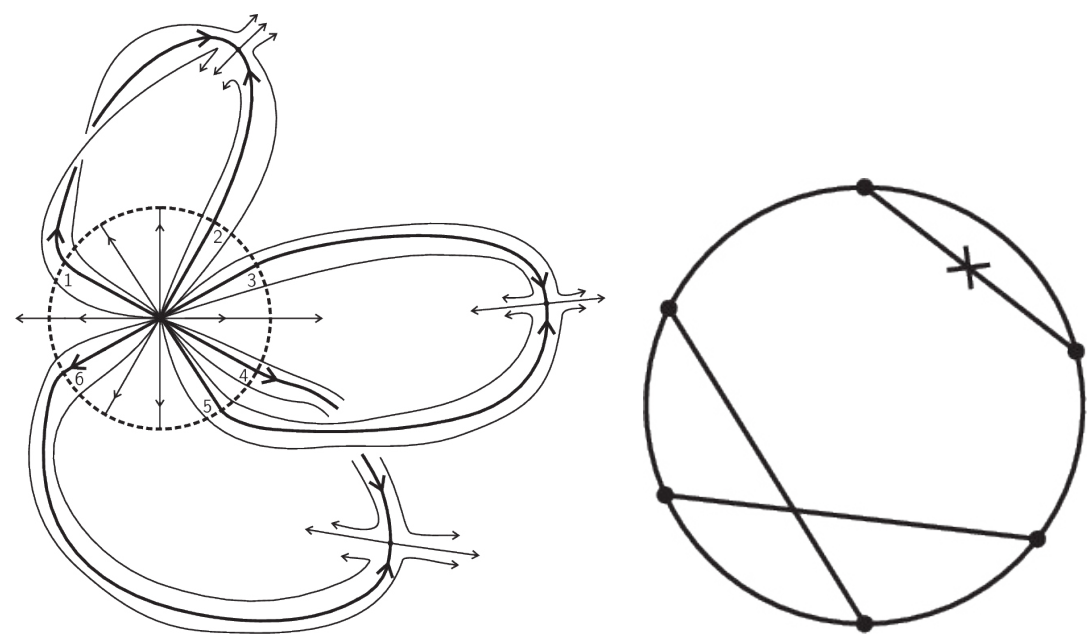

Figure 6.1. Chord diagram of a vector field

For each saddle point we consider its stable manifold consisting of two trajectories converging to that saddle. It follows from conditions 2) and 3) of the definition of Morse-Smale vector field that these trajectories start at $x_{0}$. Consequently, each of them intersects the boundary $\partial U$ at one point. Thus we get a couple of points on the boundary. The boundary $\partial U$ is a closed curve, and so it is homeomorphic to a circle $S^{1}=\left\{(x, y) \mid x^{2}+y^{2}=1\right\}$.

Via this homeomorphism the couple of points maps into a couple of points at the circle $S^{1}$. For another saddle point we will get another couple of points on $S^{1}$. Connecting the points of the same couple with the chord we will obtain chords at the circle, which do not have common ends, since the stable manifolds of the saddle points do not intersect with each other. Thus we obtain a chord diagram. If the union of $U$ with a regular neighborhood of the stable manifold is an oriented surface (homeomorphic to $S^{1} \times[0,1]$ ), then we leave chord unmarked (or formally associate to the chord the number 0 ). Otherwise (in the nonoriented case of Möbius strip), and we mark the chord by $X$ (or formally associate to the chord the number 1). Thus, each chord has a marking 0 or 1 .

Definition 6.1. A marked chord diagram is a chord diagram with marking of its chord.

A marked chord diagram constructed in such a way is a complete topological invariant of the vector field [4]. We call it a chord diagram of a vector field. 


\section{INVERSE CHORD DIAGRAM}

Let's describe a chord cycle on a chord diagram. Choose a point on a certain arc that is not the end of a chord and the direction of circumvention of a circle, for example, counterclockwise. We move from the chosen point in this direction to the nearest end of the chord. At every such point the movement on the circle changes on the chord movement. After passing the chord we continue the circular motion in counterclockwise direction if the chord is unmarked and change the direction along to clockwise if the chord is marked. We move to the nearest point that is the end of the chord. In it again change the movement on the movement of the chord. After marked chords we change the direction and after unmarked one we preserve it.
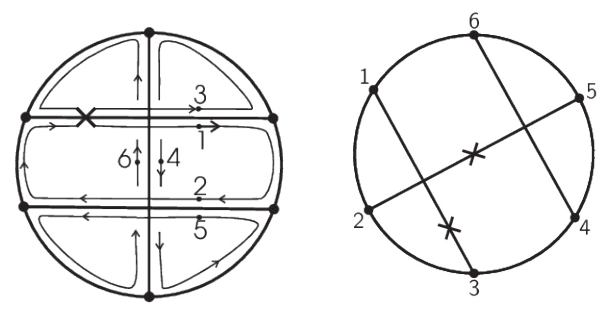

Figure 7.1. Cycle on chord diagram and inverse diagram

In the example on Figure 7.1 (left), we start from point on 11 o'clock. We then move along the higher horizontal chord 1 to its right endpoint. This chord is marked, so from its endpoint we change circle direction and move clockwise. We will continue this process until we return to the starting point.

Definition 7.1. A chord cycle is a cycle in chord diagram that obtained in a such way.

If chord cycle covers all chords (two times) and arcs we have a diagram with one chord cycle (a one-cycle diagram).

Now we construct an inverse diagram for a one-cycle diagram. On each chord we mark one point (which does not belong to other chords). When moving along the cycle we count points consecutively. Hence each point will receive two numbers, because each chord in the diagram is passed twice. We obtain a substitution of $2 n$ elements and associate to it a $2 n$-diagram in which points are connected, if their numbers correspond to the same couple in substitution, as above. By construction, each chord in the initial diagram corresponds to a chord in the inverse diagram and corresponds to a single saddle point. The chord cycle induces on each chord two directions. If these directions are opposite, then the new chord is unmarked (of marking 0). If 
these directions are same, then it is marked (of marking 1). For example, in Figure 7.1 the direction on high horizontal are same, thus, the chord $1-3$ is marked in inverse diagram.

Let us prove that the inverse chord diagram of a field $X$ is the chord diagram for the field $-X$, that is, the field has the same trajectories, but the motion behind them is changed to the opposite. Indeed, consider a regular neighborhood $W$ of the union of sources and stable manifolds of saddles. It follows from the definition of nondegenerate singular points that we can choose this neighborhood so that $X$ is transversal to the boundary $\partial W$ and directed outside it. In this case, bypassing the cycle of the chord diagram corresponds to bypassing $\partial W$. The intersections of the unstable manifolds of saddle points with $\partial W$ correspond to the chosen points on the chords and the pairs of numbers that determine the substitution. In addition, $M \backslash W$ contains one singular point, a sink. It is the source for $-X$ and $\partial W$ is the circle of the chord diagram for $-X$. The unstable manifolds of the saddles of $X$ are stable manifolds of saddles of $-X$. It completes the proof.

Consequently, the circle of the chord diagram is homeomorphic to the boundary of a regular neighborhood of the source.

From the construction of the chord diagram and the considerations above we get the following theorem.

Theorem 7.2. An optimal Morse flow on a closed surface and inverse to another flow are topologically equivalent if and only if the chord diagram of the first flow is equivalent to the inverse diagram of the other flow.

\section{EXAMPLES}

The pairs of inverse chord diagrams for polar flows on oriented surfaces of genus 1, 2 and 3 was calculated by M. Loseva, S. Tsaruk and A. Prishlyak. The number of chord in this case is even.

On torus (genus 1 ) there is unique one-cycle chord diagram with the substitution $(1,3)(2,4)$ (Figure 8.2.2 without marking).

On the surface of genus 2 there are 4 one-cycle diagrams with such substitution:

- $(1,3)(2,4)(5,7)(6,8)$ (Figure 8.6.19 without marking);

- $(1,3)(2,5)(4,7)(6,8)$ (Figure 8.6.13 without marking);

- $(1,4)(2,5)(3,7)(6,8)$ (Figure 8.6.7 without marking);

- $(1,5)(2,6)(3,7)(4,8)$ (FFigure 8.5.1-left without marking).

The inverse diagrams are the same as originals.

On the surface of genus 3 there are 82 one-cycle diagrams.30 diagrams is equivalent to their inverse. There are 26 pairs of inverse diagrams. 
We consider the case of nonorientable surfaces. The number of the chords is equal to the genus of the surface.

On the surface of genus 1 (the projective plane) there is unique diagram. It corresponds atom $\widetilde{B}$ from Bolsinov-Fomenko [1]

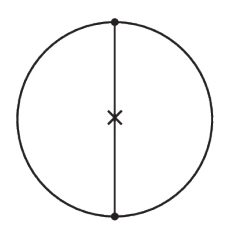

Figure 8.1. Chord diagram on the projective plane

On the surface of genus 2 (Klein bottle) there are two diagrams, see Figure 8.2. One of them corresponds to the atom $\widetilde{D}_{2}$ and the second one to $\widetilde{C}_{2}$.

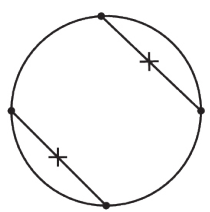

1) $\widetilde{D}_{2}$

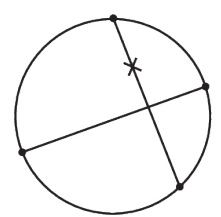

2) $\widetilde{C}_{2}$

Figure 8.2. Chord diagrams on the Klein bottle

The inverse diagrams are the same as originals.

On the nonorientable surface $N_{3}$ of genus 3 there are 7 one-cycle diagrams.

Two pairs of inverse diagrams are shown in Figure 8.3. Pair 1 correspond to atom $\widetilde{E}_{1}$ and pair 2 to $\widetilde{E}_{2}$.
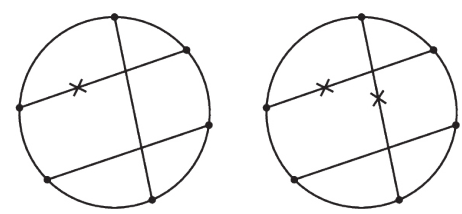

1) $\widetilde{E}_{1}$

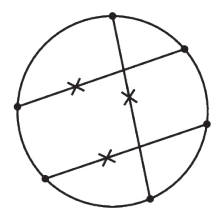

2) $\widetilde{E}_{2}$

FiguRE 8.3. Two pairs of inverse diagrams on $N_{3}$

Four selfinverse diagrams and the corresponding atoms are shown in the Figure 8.4. 


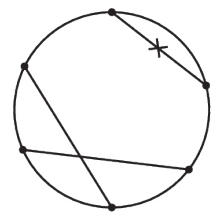

1) $\widetilde{C F}_{2}$

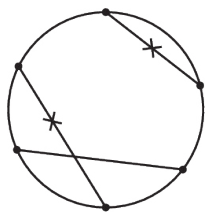

2) $\widetilde{F}_{1}$

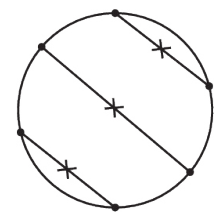

3) $\widetilde{G}_{1}$

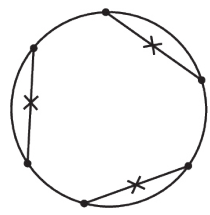

4) $\widetilde{H}_{1}$

FiguRE 8.4. 4 selfinverse diagrams on $N_{3}$

Theorem 8.1. On the nonorientable surface of genus 4 there are 47 onecycle diagrams, 12 pairs of inverse diagrams are shown in the Figure 8.5, and 23 selfinverse diagrams are shown in the Figure 8.6.

Proof. The proof consists of three parts. At first we find all chord diagrams with 4 chords. Further, we set marks on them and determine which labeled diagrams have one cycle. For every such diagram we will also find its inverse.

To list the diagrams we use lengths of their chords. The length of the chord is the minimum number of arcs of a circle necessary to pass from one end of the chord to the other along the circumference. It is one more than the number of ends of the chords being traversed in this case. For example, the diameter has a length of 4 , and the smallest chord length can be equal to 1.

If all the chords have the maximum length, then all of them are diameters. The corresponding diagram is shown in Fig. 8.5-1a (Case 1). If three chords have a length of 4 , then the fourth one will also be of length 4 , and this is the case 1 .

Assume that two chords have length 4, and the lengths of other chords are less than 4 . Then there are two options: 1) chords of length 4 perpendicular, or 2) neighboring. In the first variant the remaining two chords either have length 4 , as in the case 1 , or they have length 2 (case 2) as in Figures 8.5.1b, 8.6.1-4. In the second variant, in addition to case 1 , it is possible that the remaining chords have length 3 (case 3, Figures 8.5.2a,3a and 8.6.5) or length 1 (case 4, Figures 8.5.4a and 8.6.6).

Let one chord has length 4 , and the lengths of other chords are less than 4. Then there are three options for number of chords of length 3 :

1) 2 chords of length 3 (case 5, Figures 8.5.2b,5a,6a and 8.6.7,8);

2) 1 chord of length 3 (case 6, Figures 8.5.7a,7b,8a,9a,10a);

3 ) no chord of length 3 (case 7, Figures 8.5.5.4b and 8.6.9,10).

Further we assume that there are no chords of length 4 in the chord diagram. Then the classification is continued with respect to the number of chords of length 3 . 
Trajectory equivalence of optimal flows
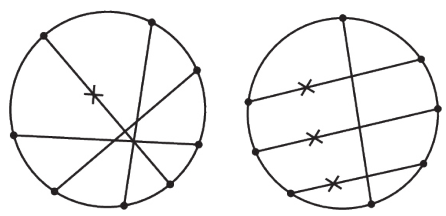

1)
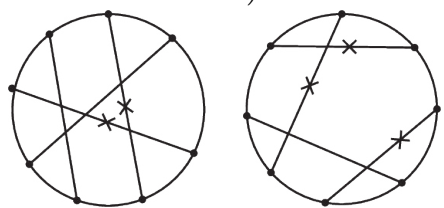

3)
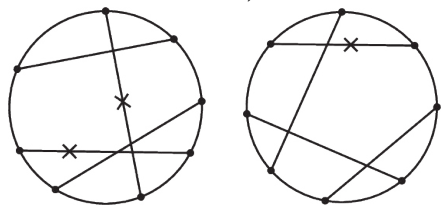

5)
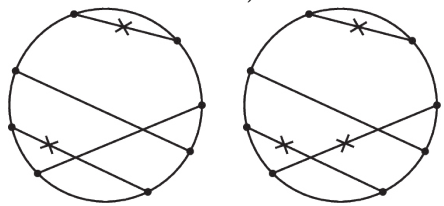

7)
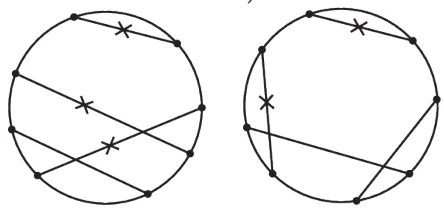

9)
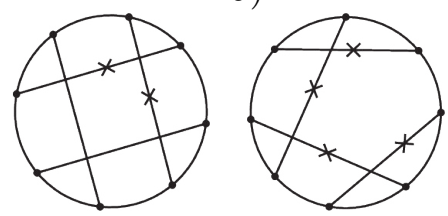

11)
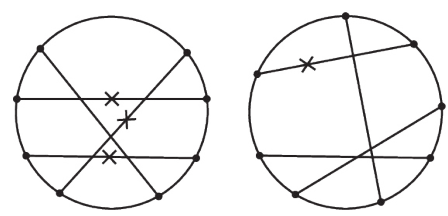

2)
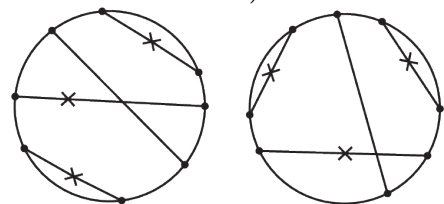

4)
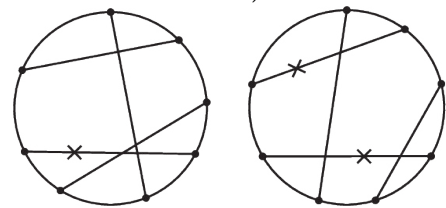

6)
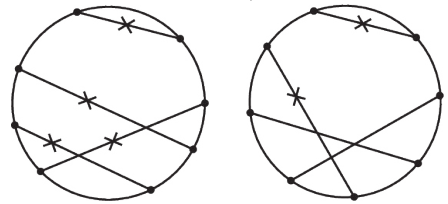

8)
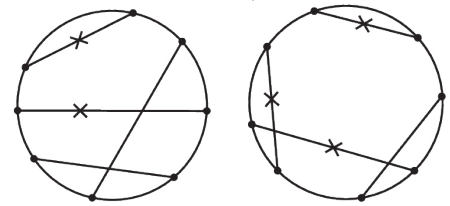

10)
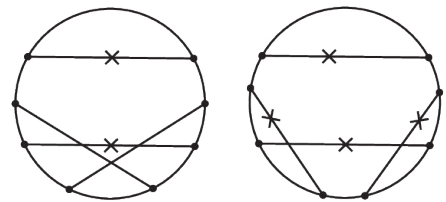

12)

FIGURE 8.5. 12 pairs of inverse diagrams on $N_{4}$

In the case of four chords of length 3, one diagram is possible (case 8, Figures 8.5.11a and 8.6.11).

In the case of three chords of length 3 , one diagram is also possible (case 9, Figures 8.5.8b,12a). 


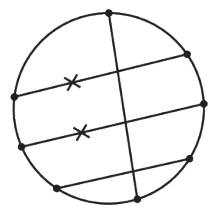

1)

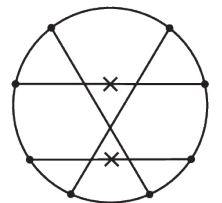

5)

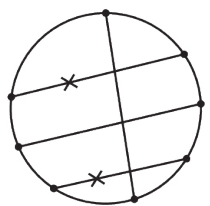

2)

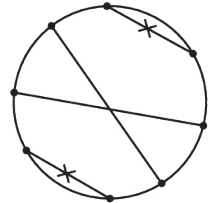

6)

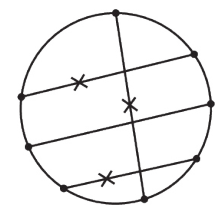

3)

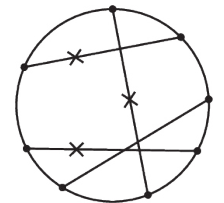

7)

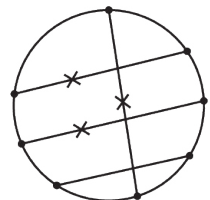

4)

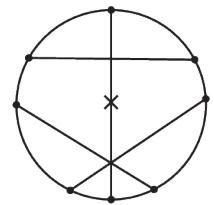

8)

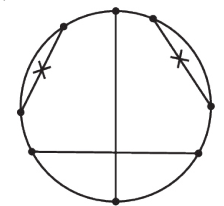

9)

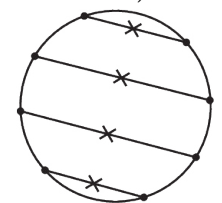

12)

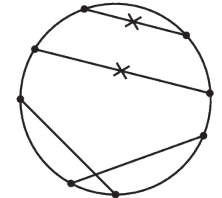

15)

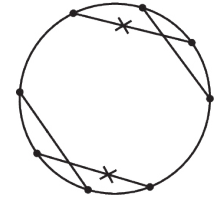

18)

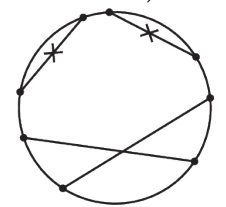

21)

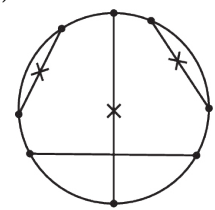

10)

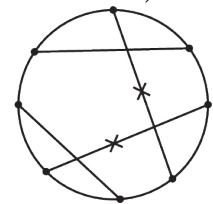

13)

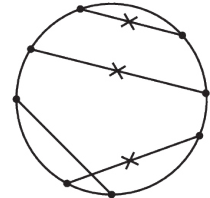

16)

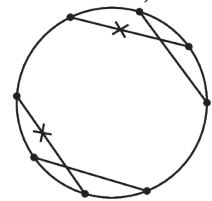

19)

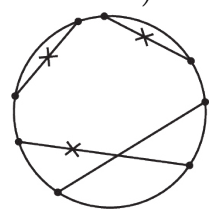

22)

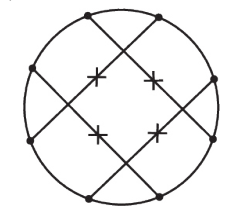

11)

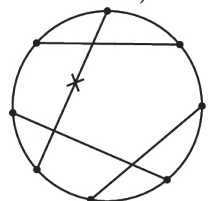

14)

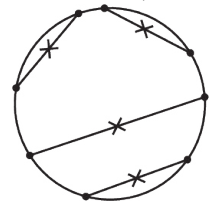

17)

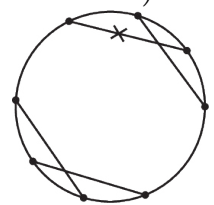

20)

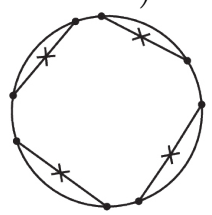

23)

FiguRe 8.6. 23 selfinverse diagrams on $N_{4}$ 
In the case of two chords of length 3, two variants are possible:

1) these chords do not intersect (case 10, Fig.8.6.12);

2) these chords intersect (case 11, Figures 8.5.3b,5b,6b,11b and 8.6.13,14).

In the case of one chord of length 3 , three variants of the number of intersections of all chords are possible:

1) two intersections (case 12, Figure 8.5.9b,10b,12b);

2) one intersection (case 13, Figure 8.6.15,16);

3) without intersections (case 14, Figure 8.6.17).

If there are no chords of lengths 3 and 4 in the diagram, then such diagrams are classified according to the number of chords of length 2 . Note that in this case chords of length 2 form pairs of intersecting chords. There are three options:

1) 4 chords of length 2 (case 15, Figure 8.6.18,19,20);

2) 2 chords of length 2 and two chords of length 1 (case 16, Figure 8.6.21,22);

3) all chords have length 1 (case 17, Figure 8.6.22).

Thus, there are 17 diagrams with four chords. On each diagram there are 15 chord markings (16th, when all chords are not marked give an oriented surface). In some diagrams, where there are symmetries, the number of markings will be smaller. Next, for each marked diagram, we find the number of cycles and leave the diagrams with one cycle. We obtained 47 marked one-cycle chord diagrams on $N_{4}$ (see Figures 8.5 and 8.6).

For them, as above, we find the inverse. As a result, we obtain 12 pairs of mutually inverse diagrams (Figures 8.5) and 23 diagrams, which are inverses to themselves (Figure 8.6).

Corollary 8.2. There are 47 topologically nonequivalent optimal Morse flows on $\mathrm{N}_{4}$ and 23 of them are topologically equivalent to reverse of themselves.

Definition 8.3. [1, p. 65] Two Morse functions $f$ and $g$ on surfaces $M$ and $N$ are called fiberwise equivalent (fiberwise frame equivalent) if there exists a diffeomorphism $h: M \rightarrow N$, which transforms the connected components of level lines of the function $f$ into those of the functions $g$ (and preserves the direction of the growth of functions).

Corollary 8.4. There are 35 fiberwise nonequivalent and 47 fiberwise frame nonequivalent Morse functions with three critical levels, one local minimum and one local maximum on $\mathrm{N}_{4}$.

Proof. Up to fiberwise equivalence, such Morse functions are given by atom, that is the restriction of functions on neighborhood of middle critical level. In this case, all possible atoms are represented as marked chords diagrams on Figures 8.5 and 8.6. 
Acknowledgements. Results of the paper are based on the talks given by Roman Shchurko at seminars of the Austro-Ukrainian Institute for Science and Technology in Vienna from November 22 till December 5, 2017, who was supported by the Project between the Austrian Academy of Sciences (AAS) and the National Academy of Sciences of Ukraine (NASU) on Fundamentals of Astroparticle and Quantum Physics.

\section{REFERENCES}

[1] A. V. Bolsinov, A. T. Fomenko. Integrable Hamiltonian systems. Geometry, Topology, Classification. A CRC Press Company, Boca Raton London New York Washington, D.C., 2004. 724 p.

[2] G. Fleitas. Classification of gradient-like flows on dimensions two and three. Bol. Soc. Brasil. Mat., 6(2):155-183, 1975.

[3] O. A. Giryk. Classification of polar Morse-Smale vector fields on two-dimensional manifolds. Methods Funct. Anal. Topology, 2(1):23 - 37, 1996.

[4] O. A. Kadubovskyj. Classification of Morse-Smale vector fields on 2-manifolds. Visn., Mat. Mekh., Kyïv. Univ. Im. Tarasa Shevchenka, (14):85-88, 2005.

[5] Y. Matsumoto. An introduction to Morse theory, volume 208 of Translations of Mathematical Monographs. American Mathematical Soc., 2002.

[6] A. A. Oshemkov, V. V. Sharko. Classication of Morse-Smale flows on two-dimensional manifolds. Mat. Sbornik, 189(8):93-140, 1998.

[7] Jacob Palis, Welington de Melo. Geometric theory of dynamical systems. An introduction. Springer-Verlag, New York-Berlin,, 1982. xii +198 p.

[8] Jacob Palis, Stephen Smale. Structural stability theorems. Global Analysis (Proc. Sympos. Pure Math., Vol. XIV, Berkeley, Calif., 1968), 1970.

[9] M. M. Peixoto. On the classication of flows of 2-manifolds. Dynamical Systems (Proc. Symp. Univ. of Bahia, Salvador, Brasil, 1971), 389-419, 1973.

[10] M.M. Peixoto. Structural stability on two-dimensional manifolds. i. Topology, 1(2):101120,1962 .

[11] Stephen Smale. On gradient dynamical systems. Ann. of Math., 74:199-206, 1961.

Received: December 18, 2017, accepted: February, 20, 2018.

Zlata Kibalko

TARAS SheVChenko University of KyIV

Email: kibakibba@gmail.com

Alexandr Prishlyak

TARAs SheVChenko University OF Kyiv

Email: prishlyak@yahoo.com

Roman Shchurko

Taras Shevchenko University of Kyiv

Email: rschurko1909@gmail.com 